durch Ambient Intelligence unter zur Grundlegung einer critical everyday life perspective (S. 159) widmet. Dabei geht es um die Herausforderungen, die durch die allgegenwärtige und gleichzeitig unsichtbare Präsenz von Technologie und deren Wirkungen auf menschliches Handeln und Verhalten entstehen.

Der letzte Hauptteil des Buches setzt sich mit methodologischen Fragen und den politischen Folgerungen und Empfehlungen aufgrund der in den Studien präsentierten Forschungsergebnisse auseinander.

Dieses Buch ist allen zu empfehlen, die sich mit IKTs und deren gesellschaftlichen Wechselbeziehungen aus einer interdisziplinären und europäischen Perspektive auseinander setzen wollen. Es hebt sich positiv dadurch hervor, dass es einerseits durchaus kritisch gegenüber technodeterministischen Strömungen in der sozialwissenschaftlichen IKT-Forschung Stellung bezieht, andererseits auf einer wohltuenden Technologiekompetenz der AutorInnen beruht. Neben den genannten AutorInnen finden sich in dem Buch weitere Beiträge von Roger Silverstone; Kees Brants und Valerie Frissen; Yves Punie, Marc Bogdanowicz, AnneJorunn Berg, Caroline Pauwels und Jean-Claude Burgelman; Francois Pichault, Dorothée Durieux und Roger Silverstone; Paschal Preston sowie von Roger Silverstone und Knut H. Sorensen.

\section{Ursula Maier-Rabler}

\section{Christa-Maria Ridder / Wolfgang R. Lan- genbucher / Ulrich Saxer / Christian Stei- ninger (Hrsg.)}

\section{Bausteine einer Theorie des öffentlich-recht- lichen Rundfunks}

Wiesbaden: VS Verlag für Sozialwissenschaften, 2005. - 472 S.

ISBN 3-531-14388-3

Fragen der Existenz, der Funktionsweise, Regulierung und Zukunft des öffentlichen Rundfunks sind seit langen Jahren ein wesentliches Forschungsgebiet der wissenschaftlichen Kommunikationspolitik. Insbesondere im internationalen Rahmen liegt dazu eine umfassende Forschungsliteratur auf zum Teil hohem theoretischen Niveau vor. In Deutschland ist diese Debatte lange - aufgrund der starken Position des Bundesverfassungsgerichtes bei der Ausge- staltung der Rundfunkordnung - von medienrechtlicher Seite aus dominiert worden. Umfassendere gesellschaftstheoretische Einordnungen sind indes bislang unterblieben. Diese Lücke schließt jetzt ein Band, der als Festschrift für Marie-Luise Kiefer herausgegeben worden ist. Christa-Maria Ridder, Wolfgang R. Langenbucher, Ulrich Saxer und Christian Steininger versammeln Autoren aus verschiedenen kommunikationswissenschaftlichen Disziplinen, die vielfältige Dimensionen und Fragestellungen der Thematik im Schnittfeld von Medienreflexion und Medienpolitik behandeln.

Besonderes Verdienst gebührt hierbei Ulrich Saxer, der in einem einleitendem Kapitel Bauvorhaben, Bausteine und Rohbau einer Theorie des öffentlich-rechtlichen Rundfunks entwirft. Seine Baustelle lässt klar erkennen, wie das fertige Gebäude einmal aussehen könnte, wobei es sich vom Gegenstand her von selbst versteht, dass die Vollendung nur ein vorübergehender Zustand sein wird, da Rundfunk im Auftrag des Gemeinwohlinteresses sich auch in $\mathrm{Zu}-$ kunft dynamisch weiterentwickelt, also auch eine Theorie schwerlich abgeschlossen sein wird.

Saxer geht in seinem Theorieentwurf systematisch vor. Er fordert, dass eine solche Theorie einem Leitkonzept folgen sollte und schlägt dafür sein Verständnis von Medien als problemlösenden und -schaffenden Systemen vor, die sich um Kommunikationskanäle institutionalisieren und organisieren. Entsprechend dem systemtheoretischen Problemverständnis spezifiziert er vier (ideale) Problemtypen für den öffentlichen Rundfunk, die eine Theorie zu berücksichtigen habe: die Wahrung seiner Identität in Übereinstimmung mit den Erwartungen, die an ihn gestellt werden im Rahmen seiner Programmaktivitäten, die Zielerreichung in Übereinstimmung mit Auflagen und wirtschaftlichen Erfordernissen, seine Anpassungsleistungen an die sich wandelnde gesellschaftliche und Medienumwelt und die Integration des gesamten Gebildes zur vollen Entfaltung seiner kommunikativen Leistungen. Im Rahmen dieser Problemkonstellation identifiziert Saxer vier Dimensionen, die auch die Strukturierung des Bandes abgeben: die rechtliche, gesellschaftliche, kommunikative und ökonomische. Während die Abgrenzung der gesellschaftlichen von der kommunikativen Dimension etwas unsauber ausfällt, gibt Saxer selbst zu, dass die Nicht-Berücksichtigung der politischen 
Dimension in diesem Band ein Mangel ist. Mit dieser Dimensionierung ist auch zugleich die Transdisziplinarität des gesamten Unterfangens eingefordert.

Weit reichend ist sein Rohbau, der Maßstäbe für eine weitere Ausformulierung einer Theorie des öffentlichen Rundfunks setzt. Sie sollte, so die Forderung, die gesellschaftliche Konstruktion, das Funktionieren und den gesellschaftlichen Impact des öffentlichen Rundfunks erklären können, und dies von der Makro-, über die Meso- bis zur Mikroebene. Die Perspektive soll dabei gerade nicht - wie es vielfältig geschieht - die Begründung der Unverzichtbarkeit des öffentlichen Rundfunks sein - dies würde dem wissenschaftlichen Grundverständnis Saxers widersprechen -, sondern den gesamten kommunikativen Lösungsbedarf und adäquate institutionelle Konstruktionen im Blick haben. Er argumentiert weiterhin konsequent funktionalistisch-systemtheoretisch, da er Rundfunk ausgehend vom Fakt, dass dieser primär eine kommunikative Funktion hat, betrachtet und des Weiteren diese Funktionalität im Hinblick auf kommunikative Bedarfslagen und Akteure und im Rückgriff auf Problemkonstellationen analysiert sehen will.

Konstituierung, Funktionalität und Regulierung sind dementsprechend die drei Bestandteile einer Theorie, die zu entwickeln wären. Würde eine Konstituierungstheorie eine Strukturanalyse des öffentlichen Rundfunks liefern, so könnte darauf aufbauend eine Funktionalitätstheorie sich der Prozessanalyse widmen und eine Regulierungstheorie (schon in einem medienpolitischem Anwendungsdiskurs) eine Funktionalitätsoptimierung des öffentlichen Rundfunks liefern. Letztendlich geht es Saxer darum, ein gemeinsames Verständnis dessen zu entwickeln, was mit dem Konzept „öffentlicher Rundfunk“ gemeint sein kann, wie er funktioniert und mit welchen Mitteln sein Leistungsvermögen gesteigert werden kann - es geht ihm um „die Einheit des Gegenstandes öffentlicher Rundfunk“.

Die ist im vorliegenden Band nicht unbedingt gegeben. Bei dem notwendig heterogenen Verfahren, das der Herausgabe einer Festschrift vorausgeht, ist nicht verwunderlich, dass die Bausteine, die von den verschiedenen Autoren herbeigetragen werden, von unterschiedlicher Passgenauigkeit für das gesamte Bauvorhaben sind. Dies ist auch in der vorliegenden Schrift der Fall. Dennoch wird in der Zusammenschau der Beiträge, insbesondere ihrer Gruppierung in den genannten vier Dimensionen, deutlich, wie viele Teilbaustellen schon weit gediehen sind.

So finden sich unter dem Kapitel der rechtlichen Dimension die Überlegungen von Martin Stock zur Funktionsgerechtigkeit des dualen Systems, in denen er die an gesellschaftlicher Rückbindung orientierte regulierte Selbstregulierung als einen geeigneten Schritt zur Erhöhung dieser Funktionalität würdigt. Für Martin Eifert sind die entsprechenden neuen Ansätze, die sich vor allem in den so genannten Selbstverpflichtungserklärungen manifestieren, nachgerade notwendiger Bestandteil der Rundfunkfreiheit auf dem Weg ihrer Weiterentwicklung. Dass Vielfalt als Leitbild medienpolitischer Aktivitäten nicht allein ökonomisch - im Hinblick auf die Bekämpfung von Medienkonzentration -, sondern auch organisationsrechtlich auszuargumentieren ist, legt Friedrich Kübler dar. Die Einbettung des Themas in den Kontext des neuen EU-Verfassungsvertrages durch Dieter Dörr ist angesichts der Bedeutung der Entscheidungen auf europäischer Ebene für den nationalen Rundfunk ebenso erhellend wie unerlässlich, auch wenn der politische Prozess in dieser Hinsicht zurzeit stagniert.

Die gesellschaftliche Dimension einer Theorie des öffentlichen Rundfunks wird in dem vorliegenden Band unter anderem durch Heinz Bonfadelli und Werner Meier ausgeführt. Sie analysieren, wie die Annahmen der aktuellen Gesellschaftskonzeptionen, die derzeit in der Kommunikationswissenschaft diskutiert werden - Wissensgesellschaft, Informationsgesellschaft, Mediengesellschaft -, Auswirkungen auf Situation und Perspektive des öffentlichen Rundfunks haben. Damit wird eine wesentliche Forderung Saxers, nämlich die nach Beachtung des kommunikativen Lösungsbedarfes, erklärungsreich beantwortet.

Selten berücksichtigt, jedoch für eine Gesamtschau der Funktionalität des öffentlichen Rundfunks unverzichtbar, ist die Darstellung der medientechnischen Innovationen, an denen er maßgeblich beteiligt war, die Albrecht Ziemer vornimmt. Der Beitrag von Christa Ridder, der eine Quote, Kosten und Qualität umfassende Kosten-Nutzen-Betrachtung des öffentlich-rechtlichen Rundfunks vornimmt, liegt zwar eher auf der Ebene des Legitimationsdiskurses, den Saxer letztlich für nicht ausreichend 
hält, liefert aber dennoch wichtige empirische Erkenntnisse zu einer Leistungsbetrachtung.

Überfällig ist sicher die Klärung des Öffentlichkeitsbegriffes für eine Theorie des öffentlichen Rundfunks - haben wesentliche Öffentlichkeitstheorien doch Medien und zudem den öffentlichen Rundfunk eher marginal behandelt. Dass sich aus solcher Klärung normative Ansprüche an diese Organisationsform des Rundfunks ableiten lassen, zeigt der Beitrag von Christian Steininger nachdrücklich. Die Möglichkeit kommunikativer Leistungen, die hier eingefordert werden, beurteilt Barbara Pfetsch in ihrer kritischen Bestandsaufnahme des Verhältnisses von öffentlich-rechtlichem Rundfunk und politischer Kommunikation im Angesicht der Synchronisierung von Medienlogik und politischer Logik mehr als skeptisch. Udo-Michael Krüger liefert zum Abschnitt der kommunikativen Dimension empirisches Material über die Politikangebote fünf großer Fernsehsender, und damit stellen diese drei Beiträge eine sehr gut aufeinander bezogene Untereinheit in dem Band dar.

Die Zuordnung der Beiträge von Schulz/Ihle und Runar Woldt in diesem Abschnitt irritiert etwas. Schulz/Ihles empirische Überprüfung vom Zusammenhang von Wettbewerb und Vielfalt am deutschen Fernsehmarkt hätte angesichts des starken medienökonomischen Bezuges besser dem entsprechenden Abschnitt angegliedert werden sollen. Und Woldts ergiebige Überblicksdarstellung zur vergleichenden Forschung zum öffentlichen Rundfunk sperrt sich ohnehin gegen das vorgegebene Strukturierungsprinzip des Bandes. Er beschreibt das Leistungsvermögen der international vergleichenden Forschung zum öffentlichen Rundfunk, das allerdings noch nicht kumuliert worden sei - eine Aufgabe, die man zumindest im Ansatz in einer Schrift zu einer Theorie des öffentlich-rechtlichen Rundfunks gerne gesehen hätte.

Bereichernd für den gesamten Band sind die Beiträge aus ökonomischer Sicht, die einige lieb gewordene Postulate des Diskurses zum öffentlich-rechtlichen Rundfunk kritisch aus der Perspektive der Medienökonomik hinterfragen - so der zur Funktionalität des Wettbewerbs (Jürgen Heinrich) oder zur Konsumentensouveränität (Manfred Kops), die in einem diskursiv gegenübergestellten Beitrag von Wolfgang Seufert indirekt wiederum bemüht wird. Der sehr weit ausholende Beitrag von Manfred
Knoche öffnet den Blick zum Abschluss noch einmal darauf, worum es bei der theoretischen Befassung mit öffentlich-rechtlichem Rundfunk im Kern geht: um eine kritische Erarbeitung der Bedingungen, unter denen die Herstellung einer demokratischen Öffentlichkeit gelingen kann - oder auch nicht.

Die Beiträge leisten, wenn auch noch nicht die Elemente für eine Theorie des öffentlichrechtlichen Rundfunks bereitzustellen - das wäre angesichts ihrer Heterogenität sowohl was Fragestellung, Ausrichtung und Zielsetzung angeht, zu viel erwartet - ein Auffächern der ganzen Breite der Problematik und Themenstellungen, die die theoretische Erfassung dieser Rundfunkorganisation erfordert. Das ist wesentlich mehr, als eine Festschrift oft erbringen kann. Auf der anderen Seite findet in vielen der Beiträge auch immer wieder eine konstruktive Rückbindung an das Euvre der Jubilarin statt, was eine gelungene Konsistenz jenseits des Gegenstandes herstellt.

Äußerst reizvoll - wenn auch nicht näher mit dem Theoriediskurs der vorangegangenen Beiträge verbunden - sind die Stellungnahmen am Ende des Bandes, die von namhaften Medienakteuren zur Bedeutung der Quote im öffentlichrechtlichen Rundfunk eingeholt wurden.

Eingangs wurde der internationale Stand der Forschung zum Public Service Broadcasting erwähnt. Für den Entwurf einer Theorie des öffentlichen Rundfunks, wie er hier vorgelegt wurde, wäre es verdienstvoll gewesen, auch die Erkenntnisse, die daraus resultieren, fruchtbar $\mathrm{zu}$ machen. Dies ist hier - außer in dem genannten Beitrag von Runar Woldt - unterblieben. Vielleicht hätte eine solche umfängliche Sichtung den Rahmen dieses Bandes gesprengt. Notwendig aber ist sie. Denn die Theorie des öffentlichen Rundfunks ist - wie gesagt - erst eine Baustelle.

Barbara Thomaß

\section{Kai Hafez}

\section{Mythos Globalisierung}

Warum die Medien nicht grenzenlos sind

Wiesbaden: VS Verlag für Sozialwissenschaften, $2005-252 \mathrm{~S}$.

ISBN 3-531-14670-X

Das ist so eine Sache mit der Globalisierung. Noch bis vor wenigen Jahren war man fest da- 